\title{
A class of exact, periodic solutions of nonlinear envelope equations
}

\author{
Kwok W. Chow ${ }^{\text {a),b) }}$ \\ Department of Mathematics, University of Arizona, Tucson, Arizona 85721
}

(Received 17 Fcbruary 1995; accepted for publication 14 April 1995)

\begin{abstract}
A class of periodic solutions of nonlinear envelope equations, e.g., the nonlinear Schrödinger equation (NLS), is expressed in terms of rational functions of elliptic functions. The Hirota bilinear transformation and theta functions are used to extend and generalize this class of solutions first reported for NISS earlier in the literature. In particular a higher order NLS and the Davey-Stewartson (DS) equations are treated. Doubly periodic standing waves solutions are obtained for both the DSI and DSII equations. A symbolic manipulation software is used to confirm the validity of the solutions independently. (C) 1995 American Institute of Physics.
\end{abstract}

\section{INTRODUCTION}

Nonlinear envelope equations have attracted intensive attention in the last three decades, since they occur in a variety of physical applications. The nonlinear Schrödinger equation (NLS \pm ) is an important representative

$$
\begin{aligned}
& i \frac{\partial A}{\partial t}+\frac{\partial^{2} A}{\partial x^{2}}+2 A^{2} A^{*}=0 \\
& i \frac{\partial A}{\partial t}+\frac{\partial^{2} A}{\partial x^{2}}-2 A^{2} A^{*}=0
\end{aligned}
$$

It governs the evolution of a weakly nonlinear wave packet in fluids, optics, and plasma physics. In the context of nonlinear fiber optics, Eq. (1.1) [(1.2)] is the anomalous (normal) dispersion regime: bright (dark) solitons occur and the plane wave shows modulational instability (stability). Exact soliton solutions have been the natural priority, due to the elegance and their prominent position in the inverse spectral approach. Recent intensive efforts in academic, commercial, and defense establishments have reinforced such interests, since solitons now have a promising potential in signals transmission over a large distance. The periodic case of envelope equations has received much less attention, partly because the mathematics is more involved. The goal of the present work is to demonstrate that the NLS, and envelope equations in general, display an intriguing variety of periodic solutions. The obvious and elementary class comprises of a single elliptic function. Another class can be constructed from rational functions of exponential and trigonometric functions. A third class, which will be the focus of this article, consists of rational functions of elliptic functions. Furthermore, elliptic functions of different moduli are involved. The conditions on the two moduli effectively form the dispersion relation of these periodic waves. An example for the NLS was first reported in Ref. 1, using a classification scheme based on the real and imaginary parts of the exact solutions of the NLS. Our objective is to extend and generalize this class of solutions to other envelope equations as well. To be precise an example cited in Ref. 1 for Eq. (1.2) is

\footnotetext{
Telephone: (602)-621-3826; FAX: (602)-621-8322; Electronic-mail: chow@math.arizona.edu

${ }^{b)}$ Present address: Department of Mechanical Engineering, Univ. of Hong Kong, Pokfulam Road, Hong Kong.
} 


$$
\begin{gathered}
\frac{r k_{1}}{\sqrt{1+k_{1}}}\left[\frac{\operatorname{cn}\left(s t, k_{1}\right)+i \sqrt{1+k_{1}} \operatorname{sn}\left(s t, k_{1}\right) \operatorname{dn}(r x, k)}{\sqrt{1+k_{1}} \operatorname{dn}(r x, k)+\operatorname{dn}\left(s t, k_{1}\right)}\right] \exp \left(-\frac{2 i r^{2}}{1+k_{1}} t\right), \\
s=\frac{2 r^{2}}{1+k_{1}}, \quad k^{2}=\frac{2 k_{1}}{1+k_{1}} .
\end{gathered}
$$

It will first be shown that an alternative representation in terms of theta functions is

$$
\begin{gathered}
\lambda\left[\frac{\left.\theta_{2}\left(\omega t, \tau_{1}\right) \theta_{4}(\alpha x, \tau)+i \theta_{1}\left(\omega t, \tau_{1}\right) \theta_{3}(\alpha x, \tau)\right]}{\theta_{3}\left(\omega t, \tau_{1}\right) \theta_{4}(\alpha x, \tau)+\theta_{3}(\alpha x, \tau) \theta_{4}\left(\omega t, \tau_{1}\right)}\right] \exp (i \Omega t), \\
\lambda^{2}=\frac{\alpha^{2} \theta_{3}^{2}(0, \tau) \theta_{4}^{2}(0, \tau) \theta_{2}^{2}\left(0, \tau_{1}\right)}{\theta_{4}^{2}\left(0, \tau_{1}\right)}, \quad \omega=\frac{2 \alpha^{2} \theta_{3}^{2}(0, \tau) \theta_{4}^{2}(0, \tau)}{\theta_{4}^{2}\left(0, \tau_{1}\right)}, \\
\Omega=-\alpha^{2}\left(\theta_{3}^{4}(0, \tau)+\theta_{4}^{4}(0, \tau)\right), \quad \frac{\theta_{3}^{2}\left(0, \tau_{1}\right)}{\theta_{4}^{2}\left(0, \tau_{1}\right)}=\frac{\theta_{3}^{4}(0, \tau)+\theta_{4}^{4}(0, \tau)}{2 \theta_{3}^{2}(0, \tau) \theta_{4}^{2}(0, \tau)} .
\end{gathered}
$$

We employ the technique of the Hirota bilinear transformation. Although Eqs. (1.5)-(1.7) appear to be slightly more complex, the symmetry allows a systematic search for new solutions. Our accomplishment can now be explained in these steps:

(1) The exact periodic solution (1.3)-(1.4) will be rederived using theta function identities and the Hirota bilinear transformation (Sec. II). It is well known that theta functions play a key role in the periodic case. ${ }^{2,3}$ Since nearly all integrable equations possess the Hirota forms, this step is critical is broadening the applicability of the present method.

(2) A higher order NLS equation will be treated to show that this procedure is probably universal (Sec. III).

(3) The technique is extended to $(2+1)$ dimensions, in particular, the Davey-Stewartson (DS) equations, to generate a new family of doubly periodic solutions in two spatial variables (Sec. IV). We use the symbolic software MATHEMATICA to verify our new exact solutions indeed satisfy DS.

Although elliptic and theta functions have been discussed extensively in the literature, ${ }^{4-6}$ a brief account will be given here for completeness. A collection of further identities will be given in the last section. The theta functions $\theta_{n}(x), n=1,2,3,4$ and the parameters $q, \tau$ (pure imaginary) are defined by

$$
\begin{gathered}
\theta_{1}(x)=\theta_{1}(x, \tau)=2 \sum_{n=0}^{\infty}(-1)^{n} q^{(n+1 / 2)^{2}} \sin (2 n+1) x \\
=-\sum_{-\infty}^{\infty} \exp \left(\pi i \tau\left(m+\frac{1}{2}\right)^{2}+2 i\left(m+\frac{1}{2}\right)\left(x+\frac{\pi}{2}\right)\right) \\
\theta_{2}(x)=\theta_{2}(x, \tau)=2 \sum_{n=0}^{\infty} q^{(n+1 / 2)^{2}} \cos (2 n+1) x=\sum_{-\infty}^{\infty} \exp \left(\pi i \tau\left(m+\frac{1}{2}\right)^{2}+2 i\left(m+\frac{1}{2}\right) x\right) \\
\theta_{3}(x)=\theta_{3}(x, \tau)=1+2 \sum_{n=1}^{\infty} q^{n^{2}} \cos 2 n x=\sum_{-\infty}^{\infty} \exp \left(\pi i \pi m^{2}+2 i m x\right) \\
\theta_{4}(x)=\theta_{4}(x, \tau)=1+2 \sum_{n=1}^{\infty}(-1)^{n} q^{n^{2}} \cos 2 n x=\sum_{-\infty}^{\infty} \operatorname{cxp}\left(\pi i \pi m^{2}+2 i m\left(x+\frac{\pi}{2}\right)\right)
\end{gathered}
$$




$$
0<q<1, \quad q=\exp (\pi i \tau), \quad q=\exp \left(-\frac{\pi K^{\prime}}{K}\right) .
$$

$K, K^{\prime}$ are the complete elliptic integrals of the first kind. Note the minus sign in front of $\theta_{1} . \theta_{1}$ is odd while the other three are even. The zeros of $\theta_{1}, \theta_{2}, \theta_{3}, \theta_{4}$ are at $M \pi+N \pi \tau,(M+1 / 2) \pi$ $+N \pi \tau,(M+1 / 2) \pi+(N+1 / 2) \pi \tau, M \pi+(N+1 / 2) \pi \tau$, respectively, $M, N$ integers. Since $\theta_{1}, \theta_{2}$ $\left(\theta_{3}, \theta_{4}\right)$ are related by a phase shift of $\pi / 2$, there are roughly two groups of theta functions. Several theta constants occur frequently in the subsequent presentations

$$
\begin{gathered}
b_{1}=2 \theta_{3}^{2}(0, \tau) \theta_{4}^{2}(0, \tau), \quad b_{2}=2 \frac{\theta_{2}^{\prime \prime}(0, \tau)}{\theta_{2}(0, \tau)}, \quad b_{3}=\frac{\theta_{3}^{\prime \prime}(0, \tau)}{\theta_{3}(0, \tau)}+\frac{\theta_{4}^{\prime \prime}(0, \tau)}{\theta_{1}(0, \tau)}, \\
\tilde{b}_{1}=2 \theta_{3}^{2}\left(0, \tau_{1}\right) \theta_{4}^{2}\left(0, \tau_{1}\right), \quad \tilde{b}_{2}=2 \frac{\theta_{2}^{\prime \prime}\left(0, \tau_{1}\right)}{\theta_{2}\left(0, \tau_{1}\right)}, \quad \tilde{b}_{3}=\frac{\theta_{3}^{\prime \prime}\left(0, \tau_{1}\right)}{\theta_{3}\left(0, \tau_{1}\right)}+\frac{\theta_{4}^{\prime \prime}\left(0, \tau_{1}\right)}{\theta_{4}\left(0, \tau_{1}\right)}
\end{gathered}
$$

for two different, but otherwise arbitrary $\tau, \tau_{1}$. Their relationship constitutes the crucial portion of the solution process.

The classical elliptic functions can be expressed as ratios of theta functions, e.g.,

$$
\begin{gathered}
\operatorname{sn}(u)=\frac{\theta_{3}(0) \theta_{1}(z)}{\theta_{2}(0) \theta_{4}(z)}, \quad \operatorname{cn}(u)=\frac{\theta_{4}(0) \theta_{2}(z)}{\theta_{2}(0) \theta_{4}(z)}, \quad \operatorname{dn}(u)=\frac{\theta_{4}(0) \theta_{3}(z)}{\theta_{3}(0) \theta_{4}(z)}, \\
z=\frac{u}{\theta_{3}^{2}(0)}, \quad k=\frac{\theta_{2}^{2}(0)}{\theta_{3}^{2}(0)}, \quad k^{\prime}=\frac{\theta_{4}^{2}(0)}{\theta_{3}^{2}(0)}, \quad k^{2}+\left(k^{\prime}\right)^{2}=1 .
\end{gathered}
$$

The Hirota bilinear transformation of Eqs. (1.1),(1.2) is [as usual, (6.18)]

$$
A=\frac{g}{f}, \quad f=f^{*}, \quad\left(i D_{t}+D_{x}^{2}-C\right) g . f=0, \quad\left(D_{x}^{2}-C\right) f \cdot f= \pm 2 g g^{*} .
$$

A single elliptic function generates the simplest periodic solution. It is worthwhile to tabulate them here for two reasons. Although the cnoidal wave of the Korteweg de Vries equation is treated extensively in the literature, ${ }^{2,3}$ the counterpart for NLS cannot be found in most standard references. Secondly, Eqs. (1.18)-(1.26) provide a preview and justification of the use of theta functions. A high degree of symmetry is observed in the theta functions formulation but not in the elliptic function case. Auxillary identities (Sec. VI) are used to satisfy Eq. (1.17) by identifying $f$ and $g$ with simple genus one theta functions. A concise explanation regarding the choice for $f$ can readily be provided. Only nonsingular solutions will be studied here, and hence the use of $\theta_{1}, \theta_{2}$ in the denominator is rejected immediately. Since $\theta_{3}, \theta_{4}$ are related by a phase shift of $\pi / 2$, this is essentially the only choice.

(1) sn-wave, normal dispersion regime (1.2) [identities (6.14)-(6.16)]:

$$
\begin{gathered}
A=\alpha \theta_{2}(0) \theta_{3}(0) \frac{\theta_{1}(\alpha x-\omega t)}{\theta_{4}(\alpha x-\omega t)} \exp (i(p x-\Omega t)), \quad \omega=2 \alpha p, \\
\Omega=p^{2}+\alpha^{2}\left(\frac{2 \theta_{4}^{\prime \prime}(0)}{\theta_{4}(0)}-\frac{\theta_{2}^{\prime \prime}(0)}{\theta_{2}(0)}-\frac{\theta_{3}^{\prime \prime}(0)}{\theta_{3}(0)}\right)=p^{2}+\alpha^{2}\left(\theta_{2}^{4}(0)+\theta_{3}^{4}(0)\right), \\
A=r k \operatorname{sn}(r x-s t) \exp (i(p x-\Omega t)), \quad s=2 r p, \quad \Omega=p^{2}+r^{2}\left(1+k^{2}\right) .
\end{gathered}
$$

(2) cn-wave, anomalous dispersion regime (1.1): 


$$
\begin{gathered}
A=\alpha \theta_{2}(0) \theta_{4}(0) \frac{\theta_{2}(\alpha x-\omega t)}{\theta_{4}(\alpha x-\omega t)} \exp (i(p x-\Omega t)), \quad \omega=2 \alpha p, \\
\Omega=p^{2}+\alpha^{2}\left(\frac{2 \theta_{3}^{\prime \prime}(0)}{\theta_{3}(0)}-\frac{\theta_{2}^{\prime \prime}(0)}{\theta_{2}(0)}-\frac{\theta_{4}^{\prime \prime}(0)}{\theta_{4}(0)}\right)=p^{2}+\alpha^{2}\left(\theta_{4}^{4}(0)-\theta_{2}^{4}(0)\right), \\
A=r k \operatorname{cn}(r x-s t) \exp (i(p x-\Omega t)), \quad s=2 r p, \quad \Omega=p^{2}+r^{2}\left(1-2 k^{2}\right) .
\end{gathered}
$$

(3) dn-wave, anomalous dispersion regime (1.1):

$$
\begin{gathered}
A=\alpha \theta_{3}(0) \theta_{4}(0) \frac{\theta_{3}(\alpha x-\omega t)}{\theta_{4}(\alpha x-\omega t)} \exp (i(p x-\Omega t)), \quad \omega=2 \alpha p, \\
\Omega=p^{2}+\alpha^{2}\left(\frac{2 \theta_{2}^{\prime \prime}(0)}{\theta_{2}(0)}-\frac{\theta_{3}^{\prime \prime}(0)}{\theta_{3}(0)}-\frac{\theta_{4}^{\prime \prime}(0)}{\theta_{4}(0)}\right)=p^{2}-\alpha^{2}\left(\theta_{3}^{4}(0)+\theta_{4}^{4}(0)\right), \\
A=r \operatorname{dn}(r x-s t) \exp (i(p x-\Omega t)), \quad s=2 r p, \quad \Omega=p^{2}-r^{2}\left(2-k^{2}\right) .
\end{gathered}
$$

One class of exact solutions, which corresponds to a stationary solitary wave on a continuous wave background in one regime, was first established by the inverse scattering transform. ${ }^{7}$ It was subsequently rederived independently by a different method, ${ }^{8}$ and periodic solutions are obtained by interchanging trigonometric and hyperbolic functions. A typical example for Eq. (1.1) is

$$
\begin{gathered}
A=\alpha \exp \left(2 i \alpha^{2} t\right)\left(1+\frac{\lambda \exp (\Omega t)+\lambda^{*} \exp (-\Omega t)}{2(\cos p x+s \cosh \Omega t)}\right), \\
\lambda=\lambda_{r}+i \lambda_{i}, \quad \lambda_{i}=\frac{p}{\alpha}, \quad \lambda_{r}^{2}=\frac{p^{4}}{\alpha^{2}\left(4 \alpha^{2}-p^{2}\right)}, \quad s=\frac{-2 \alpha^{2} \lambda_{r}}{p^{2}}, \quad \Omega=-\frac{2 p^{2}}{s \lambda_{i}} .
\end{gathered}
$$

The restriction $4 \alpha^{2}>p^{2}$ holds. The focus of the present article is a generalization of these two classes, and involves rational expressions of elliptic functions of different moduli.

\section{THETA FUNCTIONS AND THE BILINEAR OPERATOR}

Motivated by the results in Ref. 1, we search for solutions of Eq. (1.17) of the forms

$$
\begin{gathered}
g=\left(\lambda \theta_{1}\left(\omega t, \tau_{1}\right) \theta_{3}(\alpha x, \tau)+i \mu \theta_{2}\left(\omega t, \tau_{1}\right) \theta_{4}(\alpha x, \tau)\right) \exp (i \Omega t) \\
f=\theta_{3}\left(\omega t, \tau_{1}\right) \theta_{4}(\alpha x, \tau)+\theta_{3}(\alpha x, \tau) \theta_{4}\left(\omega t, \tau_{1}\right)
\end{gathered}
$$

On applying Eqs. (6.20), (6.21) to the second bilinear component of Eq. (1.17) and collecting coefficients of $\left(\theta_{3}\left(\omega t, \tau_{1}\right)\right)^{2},\left(\theta_{4}\left(\omega t, \tau_{1}\right)\right)^{2}, \theta_{3}\left(\omega t, \tau_{1}\right) \theta_{4}\left(\omega t, \tau_{1}\right)$, one obtains

$$
\begin{aligned}
\alpha^{2}\left(b_{1}\left(\theta_{3}(\alpha x, \tau)\right)^{2}+b_{2}\left(\theta_{4}(\alpha x, \tau)\right)^{2}\right)= & C\left(\theta_{4}(\alpha x, \tau)\right)^{2}-\frac{2 \lambda^{2}\left(\theta_{4}(\alpha x, \tau)\right)^{2} \theta_{3}^{2}\left(0, \tau_{1}\right)}{\theta_{2}^{2}\left(0, \tau_{1}\right)} \\
& +\frac{2 \mu^{2}\left(\theta_{3}(\alpha x, \tau)\right)^{2} \theta_{4}^{2}\left(0, \tau_{1}\right)}{\theta_{2}^{2}\left(0, \tau_{1}\right)}
\end{aligned}
$$




$$
\begin{gathered}
\alpha^{2}\left(b_{2}\left(\theta_{3}(\alpha x, \tau)\right)^{2}+b_{1}\left(\theta_{4}(\alpha x, \tau)\right)^{2}\right)=C\left(\theta_{3}(\alpha x, \tau)\right)^{2}+\frac{2 \lambda^{2}\left(\theta_{4}(\alpha x, \tau)\right)^{2} \theta_{4}^{2}\left(0, \tau_{1}\right)}{\theta_{2}^{2}\left(0, \tau_{1}\right)} \\
-\frac{2 \mu^{2}\left(\theta_{3}(\alpha x, \tau)\right)^{2} \theta_{3}^{2}\left(0, \tau_{1}\right)}{\theta_{2}^{2}\left(0, \tau_{1}\right)}, \\
C=\alpha^{2} b_{3} .
\end{gathered}
$$

Equations (6.11), (6.12) are needed to obtain $\theta_{1}^{2}, \theta_{2}^{2}$ in terms of $\theta_{3}^{2}, \theta_{4}^{2}$. One now continues to use the concept of linear independence, and collect terms of $\left(\theta_{3}(\alpha x, \tau)\right)^{2},\left(\theta_{4}(\alpha x, \tau)\right)^{2}$, $\theta_{3}(\alpha x, \tau) \theta_{4}(\alpha x, \tau)$ in Eqs. (2.3)-(2.5). Consistency is assured if

$$
\lambda^{2}=\mu^{2}, \quad \alpha^{2} b_{1}=\frac{2 \lambda^{2} \theta_{4}^{2}\left(0, \tau_{1}\right)}{\theta_{2}^{2}\left(0, \tau_{1}\right)}, \quad \alpha^{2}\left(b_{3}-b_{2}\right)=\frac{2 \lambda^{2} \theta_{3}^{2}\left(0, \tau_{1}\right)}{\theta_{2}^{2}\left(0, \tau_{1}\right)}
$$

and hence the defining equation between $\tau$ and $\tau_{1}$ by Eqs. (6.14) $-(6.16)$ is

$$
\frac{\theta_{3}^{2}\left(0, \tau_{1}\right)}{\theta_{4}^{2}\left(0, \tau_{1}\right)}=\frac{\theta_{3}^{4}(0, \tau)+\theta_{4}^{4}(0, \tau)}{2 \theta_{3}^{2}(0, \tau) \theta_{4}^{2}(0, \tau)}
$$

Similarly we go through such a two-step $t, x$ decomposition procedure again for the first component of Eq. (1.17). On collecting terms of $\theta_{2}\left(\omega t, \tau_{1}\right) \theta_{3}\left(\omega t, \tau_{1}\right), \theta_{2}\left(\omega t, \tau_{1}\right) \theta_{4}\left(\omega t, \tau_{1}\right)$ (for the real part), $\theta_{1}\left(\omega t, \tau_{1}\right) \theta_{3}\left(\omega t, \tau_{1}\right), \theta_{1}\left(\omega t, \tau_{1}\right) \theta_{4}\left(\omega t, \tau_{1}\right)$ (for the imaginary part), one obtains the frequencies of the oscillation

$$
\Omega=\alpha^{2}\left(b_{2}-b_{3}\right), \quad \omega=\frac{\Omega}{\theta_{3}^{2}\left(0, \tau_{1}\right)}
$$

The $b_{n}$ 's are given by Eqs. (1.13), (1.14).

\section{HIGHER ORDER NLS}

NLS is typically only the leading term in the evolution of the wave packet. Higher order terms are necessary when greater accuracy is desired. In hydrodynamics, surface gravity waves of moderate steepness call for these "fourth order nonlinear equations," sometimes called the Dysthe's equation. ${ }^{9}$ In nonlinear fiber optics, studies of pulses of short duration demand the introduction of these higher order terms. ${ }^{10}$ Although such higher order NLS equations are generally not integrable, a special version exists which admits $N$ (bright) soliton solutions ${ }^{11}$

$$
i A_{t}+A_{x x}+A^{2} A^{*}+i \nu A_{x x x}+3 i \nu A A^{*} A_{x}=0 \text {. }
$$

Parallel to the analysis of Sec. II only the dark soliton regime will be considered here ( $f$ real)

$$
\begin{gathered}
i A_{t}+A_{x x}-A^{2} A^{*}+i \nu A_{x x x}-3 i \nu A A^{*} A_{x}=0 \\
A=\frac{G}{f}, \quad\left(D_{x}^{2}-C\right) f \cdot f=-G G^{*}, \quad\left(i D_{t}-3 i \nu C D_{x}+D_{x}^{2}+i \nu D_{x}^{3}-C\right) G \cdot f=0 .
\end{gathered}
$$

A simple mapping allows one to conclude

$$
G=g \exp (i(p x-\Omega t)), \quad\left(D_{x}^{2}-C\right) f . f=-g g^{*},
$$




$$
\left[i D_{t}+i\left(-3 \nu C+2 p-3 \nu p^{2}\right) D_{x}+(1-3 \nu p) D_{x}^{2}+i \nu D_{x}^{3}+\left(\Omega+3 \nu C p-p^{2}+\nu p^{3}-C\right)\right] g . f=0
$$

An analogous two-step decomposition analysis of the previous section can be performed. We again assign the selection

$$
\begin{gathered}
g=\lambda\left[\theta_{2}\left(\omega t, \tau_{1}\right) \theta_{4}(\alpha x, \tau)+i \theta_{1}\left(\omega t, \tau_{1}\right) \theta_{3}(\alpha x, \tau)\right], \\
f=\theta_{3}\left(\omega t, \tau_{1}\right) \theta_{4}(\alpha x, \tau)+\theta_{4}\left(\omega t, \tau_{1}\right) \theta_{3}(\alpha x, \tau) .
\end{gathered}
$$

The trick is to insist that the contributions from the $D_{x}$ and $D_{x}^{3}$ terms sum up to zero. $\lambda$ and the dispersion relation are still Eqs. (2.6), (2.7), but the frequencies are now

$$
\omega \theta_{4}^{2}\left(0, \tau_{1}\right)=\alpha^{2} b_{1}(1-3 \nu p), \quad \Omega-p^{2}+\nu p^{3}=\alpha^{2}\left(b_{3}-b_{2}\right)(1-3 \nu p) .
$$

One precaution must be exercised. $p$ is given by

$$
3 \nu p^{2}-2 p+2 \nu \alpha^{2}\left(\theta_{3}^{4}(0)+\theta_{4}^{4}(0)\right)=0 .
$$

Real $p$ implies the restriction

$$
\alpha^{2} \nu^{2}\left(\theta_{3}^{4}(0, \tau)+\theta_{3}^{4}(0, \tau)\right)<\frac{1}{6}
$$

\section{THE DAVEY-STEWARTSON EQUATIONS}

The Benney-Roskes, Davey-Stewartson equations (DS) govern the evolution of $(2+1)$ dimensional weakly nonlinear wave packets in hydrodynamics 9

$$
i A_{t}-A_{x x}-\sigma^{2} A_{y y}+\nu A^{2} A^{*}=2 Q A, \quad Q_{x x}-\sigma^{2} Q_{y y}=\nu\left(A A^{*}\right)_{x x} .
$$

From a theoretical perspective DS plays a fundamental role in the theory of solvable $(2+1)$ dimensional equations. $\sigma=1(\sigma=i)$ corresponds to the DSI (DSII) case. The bilinear form is $\left[A=G / f, Q=2(\log f)_{x x}, f\right.$ real, $C=$ const $]$

$$
\left(i D_{t}-D_{x}^{2}-\sigma^{2} D_{y}^{2}-C\right) G . f=0, \quad\left(D_{x}^{2}-\sigma^{2} D_{y}^{2}-C\right) f . f=\nu G G^{*} .
$$

"Doubly periodic" in the present usage means that the solution is periodic in both the $x, y$ directions. One type of traveling doubly periodic waves for the Kadomtsev-Petviashvili and DS equations was obtained earlier in the literature. ${ }^{12,13}$ Intuitively a theta function of genus two is needed to describe these doubly periodic waves. The surprising point is that, at least in one particular case here, a product of two genus one functions is sufficient. This is more than an academic interest, since genus one functions greatly reduce the ensuing analytical and computational complexity. The present article proves that a similar conclusion also prevails in the case of standing waves. In fact we are able to derive two different families of doubly periodic standing waves, one for DSI and one for DSII, using rational expressions of two genus one theta functions.

The central themes of this article, namely, theta functions and the bilinear transformation, are again employed. A little thought on the symmetry of theta function identities enables us to deduce $\operatorname{DSI}(\sigma=1)$ :

$$
\begin{gathered}
\sigma=1, \quad \nu=2, \\
G=\lambda\left[\theta_{3}(\alpha x, \tau) \theta_{3}\left(\beta y, \tau_{1}\right)+\theta_{4}(\alpha x, \tau) \theta_{4}\left(\beta y, \tau_{1}\right)\right] \exp (i \Omega t), \\
f=\theta_{3}(\alpha x, \tau) \theta_{4}\left(\beta y, \tau_{1}\right)+\theta_{4}(\alpha x, \tau) \theta_{3}\left(\beta y, \tau_{1}\right) .
\end{gathered}
$$


We undergo a two-step $x, t$ decomposition similar to that of Sec. II. The dispersion relation and the relevant parameters for Eq. (4.2) are

$$
\begin{gathered}
\alpha^{2}\left[\theta_{3}^{2}(0, \tau)-\theta_{4}^{2}(0, \tau)\right]^{2}=\beta^{2}\left[\theta_{3}^{2}\left(0, \tau_{1}\right)-\theta_{4}^{2}\left(0, \tau_{1}\right)\right]^{2} \\
C=\alpha^{2} b_{2}-\beta^{2} \tilde{b}_{2} \\
\lambda^{2}=\alpha^{2} \theta_{3}^{2}(0, \tau) \theta_{4}^{2}(0, \tau)-\beta^{2} \theta_{3}^{2}\left(0, \tau_{1}\right) \theta_{4}^{2}\left(0, \tau_{1}\right) \\
\Omega=-\alpha^{2}\left[\frac{\theta_{3}^{\prime \prime}(0, \tau)}{\theta_{3}(0, \tau)}+\frac{\theta_{4}^{\prime \prime}(0, \tau)}{\theta_{4}(0, \tau)}+\frac{2 \theta_{2}^{\prime \prime}(0, \tau)}{\theta_{2}(0, \tau)}\right]-2 \beta^{2} \theta_{3}^{2}\left(0, \tau_{1}\right) \theta_{4}^{2}\left(0, \tau_{1}\right) \\
A=\lambda\left[\frac{\theta_{3}(\alpha x, \tau) \theta_{3}\left(\beta y, \tau_{1}\right)+\theta_{4}(\alpha x, \tau) \theta_{4}\left(\beta y, \tau_{1}\right)}{\theta_{3}(\alpha x, \tau) \theta_{4}\left(\beta y, \tau_{1}\right)+\theta_{4}(\alpha x, \tau) \theta_{3}\left(\beta y, \tau_{1}\right)}\right] \exp (i \Omega t) \\
Q=\frac{R}{\left[\theta_{3}(\alpha x, \tau) \theta_{4}\left(\beta y, \tau_{1}\right)+\theta_{4}(\alpha x, \tau) \theta_{3}\left(\beta y, \tau_{1}\right)\right]^{2}} \\
\left.R=\alpha^{2} \theta_{4}^{2}\left(\beta y, \tau_{1}\right)\left(b_{2} \theta_{3}^{2}(\alpha x, \tau)+b_{1} \theta_{4}^{2}(\alpha x, \tau)\right)+\alpha^{2} \theta_{3}^{2}\left(\beta y, \tau_{1}\right)\left(b_{1} \theta_{3}^{2}(\alpha x, \tau)+b_{2} \theta_{4}^{2}(\alpha x, \tau)\right)\right] \\
+2 \alpha^{2} b_{3} \theta_{3}(\alpha x, \tau) \theta_{3}\left(\beta y, \tau_{1}\right) \theta_{4}(\alpha x, \tau) \theta_{4}\left(\beta y, \tau_{1}\right)
\end{gathered}
$$

\section{Confirmation:}

It is convenient first to transform the theta formulation into the Jacobi elliptic functions. Since the arguments of the theta and elliptic functions differ by the scalar $\theta_{3}^{2}(0)$, the new wave numbers $r, s$ are used.

$$
\begin{gathered}
r=\alpha \theta_{3}^{2}(0, \tau), \quad s=\beta \theta_{3}^{2}\left(0, \tau_{1}\right), \\
Q=-\frac{2 r^{2} E}{K}+2 r^{2}\left(\frac{\sqrt{1-k^{2}}\left(1+X^{2} Y^{2}\right)+\left(2-k^{2}\right) X Y}{(X+Y)^{2}}\right), \\
A=\lambda\left(\frac{1+X Y}{X+Y}\right) \exp (i \Omega t), \quad X=\frac{\operatorname{dn}(r x, k)}{\left(1-k^{2}\right)^{1 / 4}}, \quad Y=\frac{\operatorname{dn}\left(s y, k_{1}\right)}{\left(1-k_{1}^{2}\right)^{1 / 4}}
\end{gathered}
$$

where $K$ and $E$ are complete elliptic integrals of the first and second kind

$$
\begin{gathered}
K=\int_{0}^{\pi / 2} \frac{d \xi}{\sqrt{1-k^{2} \sin ^{2} \xi}}, \quad E=\int_{0}^{\pi / 2} \sqrt{1-k^{2} \sin ^{2} \xi} d \xi \\
r\left(1-\sqrt{1-k^{2}}\right)=s\left(1-\sqrt{1-k_{1}^{2}}\right), \quad \lambda^{2}=r^{2} \sqrt{1-k^{2}}-s^{2} \sqrt{1-k_{1}^{2}} \\
-\Omega=-\frac{4 r^{2} E}{K}+2 r^{2} \sqrt{1-k^{2}}+s^{2}\left(2-k_{1}^{2}\right)
\end{gathered}
$$

The symbolic manipulation software MATHEMATICA is now used to verify that Eqs. (4.3) and (4.14)-(4.18) indeed solve Eq. (4.1). A remark regarding the actual implementation is in order. Though $k$ is used to denote the modulus here, Jacobi functions in MATHEMATICA require an input $m$. The relationship $m=k^{2}$ holds. $Q$ and $A$ are illustrated in Figs. 1 and 2 . 


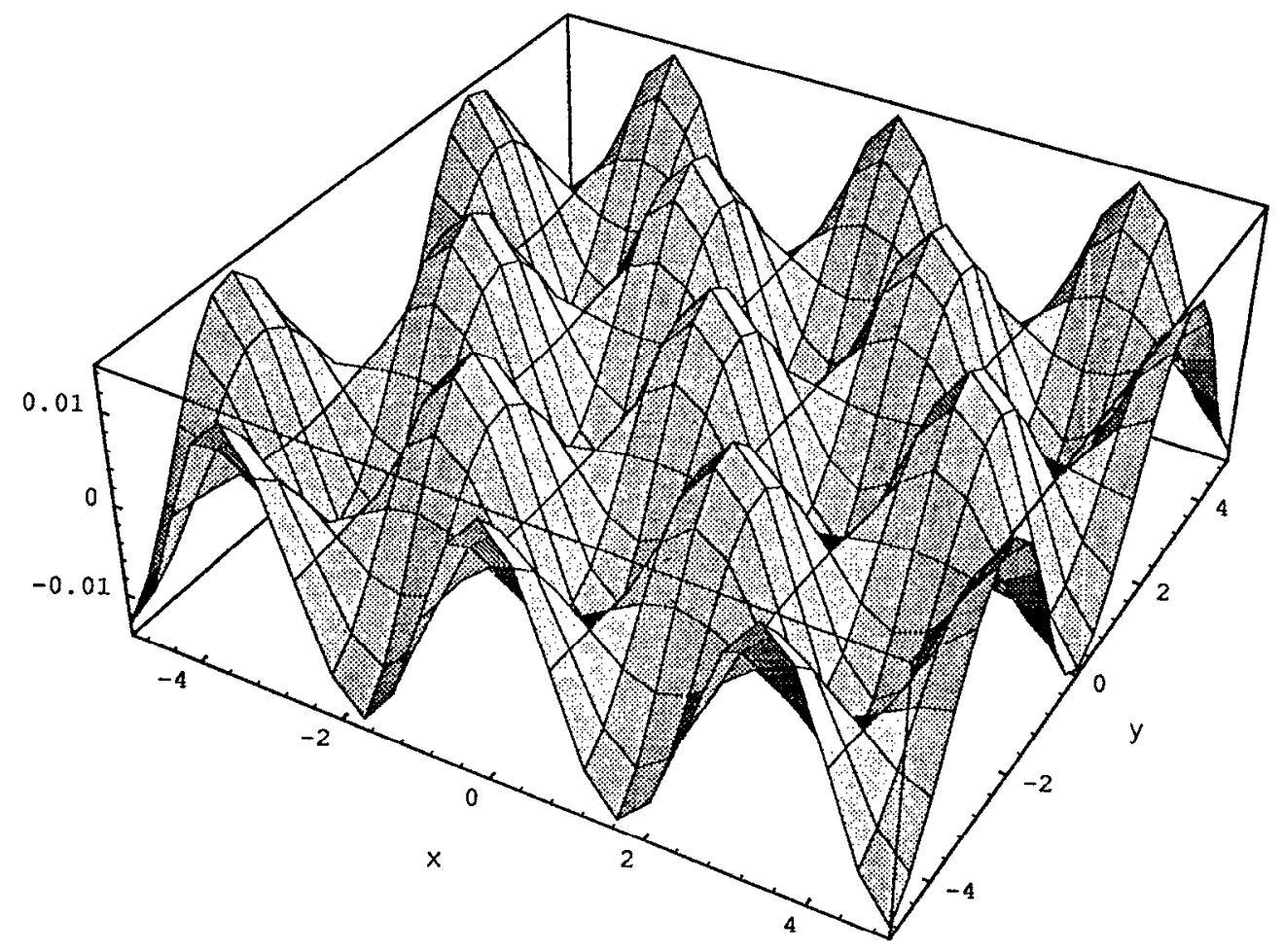

FIG. 1. The mean flow $Q$ of Eqs. (4.13)-(4.18) vs $x, y$, from -5 to $5 . k=0.5, k_{1}=0.6, r=1$.

$\operatorname{DSII}(\sigma=i)$ :

$$
\begin{gathered}
\sigma=i, \quad \nu=-2, \\
G=\lambda\left[\theta_{1}(\alpha x, \tau) \theta_{2}\left(\beta y, \tau_{1}\right)+i \theta_{2}(\alpha x, \tau) \theta_{1}\left(\beta y, \tau_{1}\right)\right] \exp (i \Omega t), \\
f=\theta_{3}(\alpha x, \tau) \theta_{4}\left(\beta y, \tau_{1}\right)+\theta_{4}(\alpha x, \tau) \theta_{3}\left(\beta y, \tau_{1}\right)
\end{gathered}
$$

The dispersion relation and the relevant parameters for Eq. (4.2) are

$$
\begin{gathered}
\alpha^{2} \theta_{2}^{4}(0, \tau)=\beta^{2} \theta_{2}^{4}\left(0, \tau_{1}\right) \\
C=\alpha^{2} b_{3}+\beta^{2} \tilde{b}_{3} \\
2 \alpha^{2} \theta_{3}^{2}(0, \tau) \theta_{4}^{2}(0, \tau)+2 \beta^{2} \theta_{3}^{2}\left(0, \tau_{1}\right) \theta_{4}^{2}\left(0, \tau_{1}\right)=\frac{2 \lambda^{2}\left(\theta_{3}^{2}(0, \tau) \theta_{4}^{2}\left(0, \tau_{1}\right)+\theta_{3}^{2}\left(0, \tau_{1}\right) \theta_{4}^{2}(0, \tau)\right)}{\theta_{2}^{2}(0, \tau) \theta_{2}^{2}\left(0, \tau_{1}\right)} \\
\Omega=-\alpha^{2}\left[\frac{\theta_{2}^{\prime \prime}(0, \tau)}{\theta_{2}(0, \tau)}+\frac{\theta_{4}^{\prime \prime}(0, \tau)}{\theta_{4}(0, \tau)}+\frac{2 \theta_{3}^{\prime \prime}(0, \tau)}{\theta_{3}(0, \tau)}\right]-\beta^{2} \theta_{3}^{4}\left(0, \tau_{1}\right) \\
A=\lambda\left[\frac{\theta_{1}(\alpha x, \tau) \theta_{2}\left(\beta y, \tau_{1}\right)+i \theta_{2}(\alpha x, \tau) \theta_{1}\left(\beta y, \tau_{1}\right)}{\theta_{3}(\alpha x, \tau) \theta_{4}\left(\beta y, \tau_{1}\right)+\theta_{4}(\alpha x, \tau) \theta_{3}\left(\beta y, \tau_{1}\right)}\right] \exp (i \Omega t)
\end{gathered}
$$




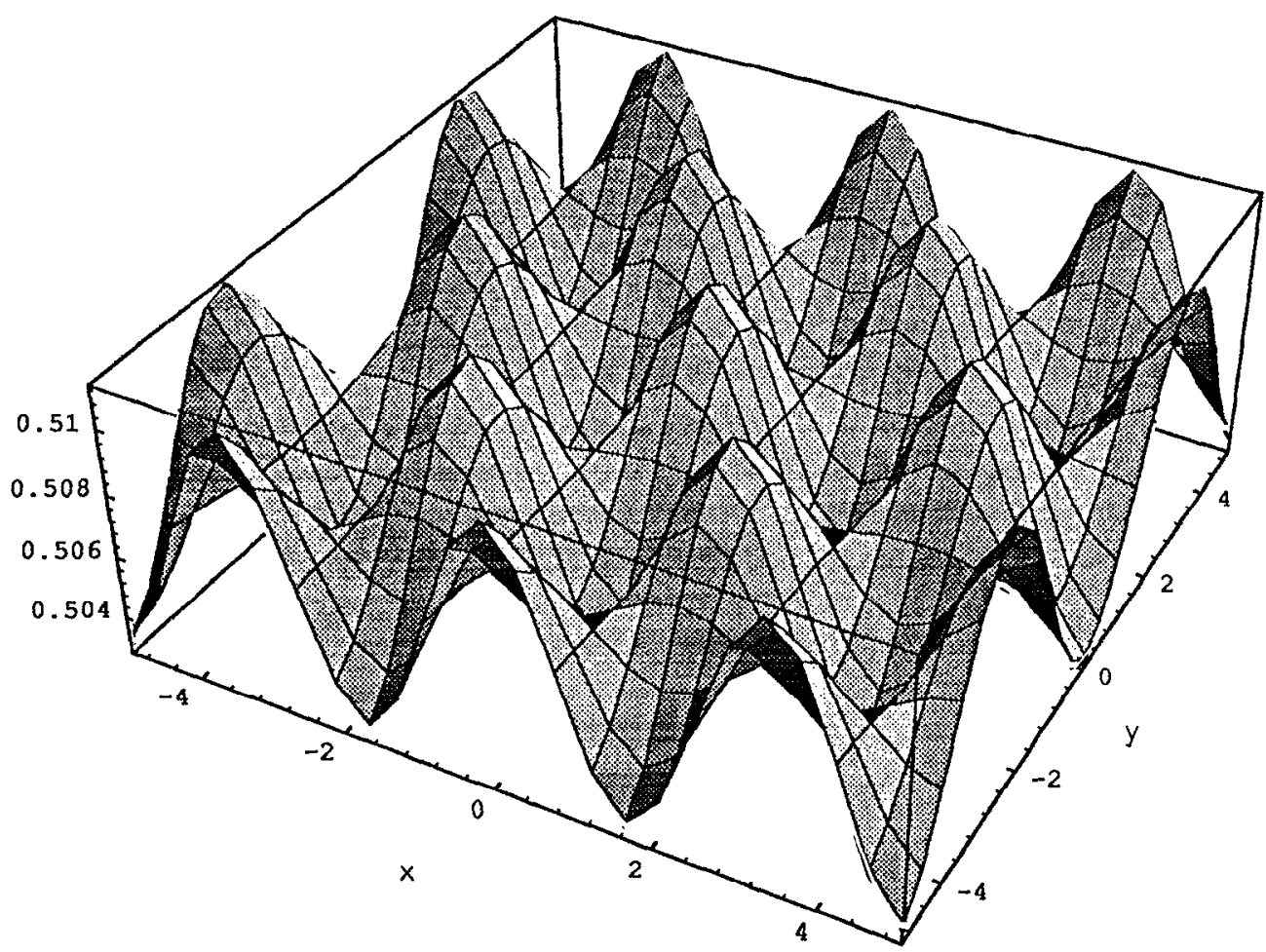

FIG. 2. Modulus $A$ square, $|A|^{2}$, Eqs. (4.13) $-(4.18)$ vs $x, y$, from -5 to $5 . k=0.5, k_{1}=0.6, r=1$.

$$
\begin{gathered}
Q=\frac{R}{\left[\theta_{3}(\alpha x, \tau) \theta_{4}\left(\beta y, \tau_{1}\right)+\theta_{4}(\alpha x, \tau) \theta_{3}\left(\beta y, \tau_{1}\right)\right]^{2}} \\
\left.R=\alpha^{2} \theta_{4}^{2}\left(\beta y, \tau_{1}\right)\left(b_{2} \theta_{3}^{2}(\alpha x, \tau)+b_{1} \theta_{4}^{2}(\alpha x, \tau)\right)+\alpha^{2} \theta_{3}^{2}\left(\beta y, \tau_{1}\right)\left(b_{1} \theta_{3}^{2}(\alpha x, \tau)+b_{2} \theta_{4}^{2}(\alpha x, \tau)\right)\right] \\
+2 \alpha^{2} b_{3} \theta_{3}(\alpha x, \tau) \theta_{3}\left(\beta y, \tau_{1}\right) \theta_{4}(\alpha x, \tau) \theta_{4}\left(\beta y, \tau_{1}\right)
\end{gathered}
$$

\section{Confirmation:}

The elliptic function version of the solution for DSII is

$$
\begin{gathered}
r=\alpha \theta_{3}^{2}(0, \tau), \quad s=\beta \theta_{3}^{2}\left(0, \tau_{1}\right), \quad X=\frac{\operatorname{dn}(r x, k)}{\left(1-k^{2}\right)^{1 / 4}}, \quad Y=\frac{\operatorname{dn}\left(s y, k_{1}\right)}{\left(1-k_{1}^{2}\right)^{1 / 4}}, \\
Q=-\frac{2 r^{2} E}{K}+2 r^{2}\left(\frac{\sqrt{1-k^{2}}\left(1+X^{2} Y^{2}\right)+\left(2-k^{2}\right) X Y}{(X+Y)^{2}}\right), \\
A=\lambda \sqrt{k k_{1}}\left(\frac{A_{1}}{A_{2}}\right) \exp (i \Omega t), \\
A_{1}=\frac{\operatorname{sn}(r x, k) \operatorname{cn}\left(s y, k_{1}\right)}{\left(1-k_{1}^{2}\right)^{1 / 4}}+i \frac{\operatorname{cn}(r x, k) \operatorname{sn}\left(s y, k_{1}\right)}{\left(1-k^{2}\right)^{1 / 4}}
\end{gathered}
$$




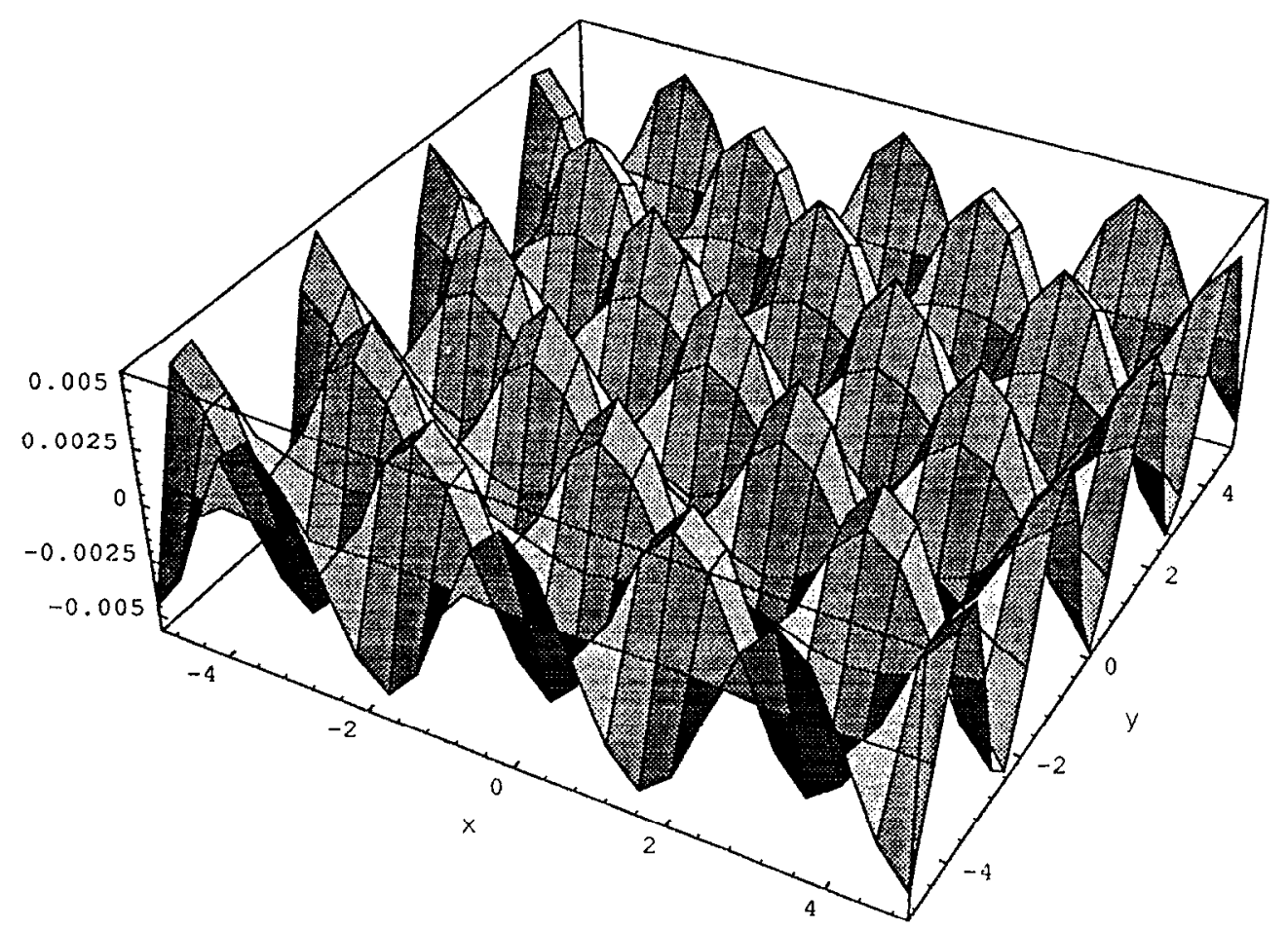

FIG. 3. The mean flow $Q$ of Eqs. (4.29)-(4.35) vs $x, y$, from -5 to $5 . k=0.5, k_{1}=0.4, r=1$.

$$
\begin{gathered}
A_{2}=\frac{\operatorname{dn}(r x, k)}{\left(1-k^{2}\right)^{1 / 4}}+\frac{\operatorname{dn}\left(s y, k_{1}\right)}{\left(1-k_{1}^{2}\right)^{1 / 4}}, \\
r k=s k_{1}, \\
\lambda^{2}=\frac{k k_{1}\left(r^{2} \sqrt{1-k^{2}}+s^{2} \sqrt{1-k_{1}^{2}}\right)}{\sqrt{1-k^{2}}+\sqrt{1-k_{1}^{2}}}, \quad \Omega=\frac{4 r^{2} E}{K}-r^{2}\left(3-2 k^{2}\right)-s^{2},
\end{gathered}
$$

where $E, K$ are given by Eq. (4.16). MATHEMATICA is again used to verify Eqs. (4.19) and (4.29)(4.35) satisfy Eq. (4.1). To reduce complexity it is best to verify the real and imaginary parts of Eq. (4.1) separately. Note that $G$ is purely real in DSI but complex in DSII. $Q$ and $A$ are illustrated in Figs. 3 and 4.

\section{CONCLUSIONS}

A class of periodic solutions of nonlinear envelope equations is developed, using a higher order NLS and DS as examples. The procedure is probably applicable to other evolution systems of physical interests, e.g., the Zakharov equations in plasma physics, the long wave-short wave resonance equation in water waves. ${ }^{14}$ We stress that theta functions provide a high degree of symmetry in the formulation, and enhance the ease in the search for new solutions. However, the Jacobi elliptic functions yield a slightly more compact answer, and are readily available for usage in a symbolic software package. Apparently NLS and related envelope equations display a much 


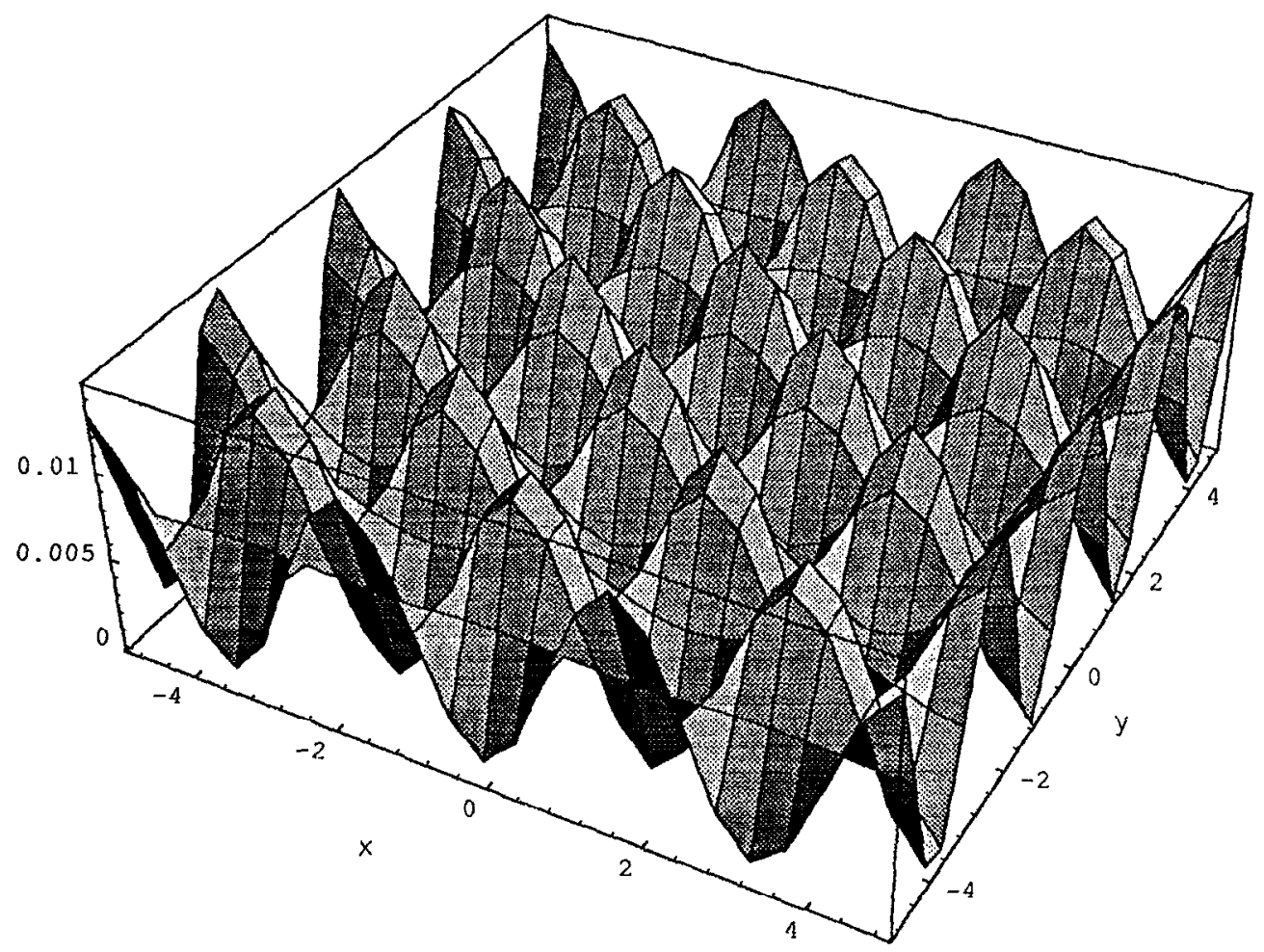

FIG. 4. Modulus $A$ square, $|A|^{2}$, Eqs. (4.29)-(4.35) vs $x, y$, from -5 to $5 . k=0.5, k_{1}=0.4, r=1$.

richer variety of periodic solutions than the celebrated Korteweg de Vries (KdV) equation. Many problems remain. We have been unable to find similar periodic solutions in the anomalous dispersion regime $[N L S+,(1.1)]$. Another question is connected with the superposition of solitons and the periodic solution. An infinite sum of regularly spaced $\mathrm{KdV}$ solitons gives an exact solution of $\mathrm{KdV}^{3}$ A similar summation process for the NLS does not reproduce any of the periodic exact solutions mentioned in this article. The error is at least an exponential phase factor in time. ${ }^{15} \mathrm{We}$ do not know how to resolve the puzzle. The relation between the present class of solutions and the inverse spectral transform is unclear. The geometric structure of these periodic solutions remains unexplored. ${ }^{16}$ More exciting discoveries still remain ahead.

\section{THETA FUNCTIONS IDENTITIES}

There exists a large class of identities involving products of theta functions, for example,

$$
\begin{gathered}
\theta_{3}(x+y) \theta_{3}(x-y) \theta_{2}^{2}(0)=\theta_{4}^{2}(x) \theta_{1}^{2}(y)+\theta_{3}^{2}(x) \theta_{2}^{2}(y), \\
\theta_{4}(x+y) \theta_{4}(x-y) \theta_{2}^{2}(0)=\theta_{4}^{2}(x) \theta_{2}^{2}(y)+\theta_{3}^{2}(x) \theta_{1}^{2}(y), \\
\theta_{1}(x+y) \theta_{2}(x-y) \theta_{3}(0) \theta_{4}(0)=\theta_{1}(x) \theta_{2}(x) \theta_{3}(y) \theta_{4}(y)+\theta_{3}(x) \theta_{4}(x) \theta_{1}(y) \theta_{2}(y), \\
\theta_{1}(x+y) \theta_{3}(x-y) \theta_{2}(0) \theta_{4}(0)=\theta_{1}(x) \theta_{3}(x) \theta_{2}(y) \theta_{4}(y)+\theta_{2}(x) \theta_{4}(x) \theta_{1}(y) \theta_{3}(y), \\
\theta_{1}(x+y) \theta_{4}(x-y) \theta_{2}(0) \theta_{3}(0)=\theta_{1}(x) \theta_{4}(x) \theta_{2}(y) \theta_{3}(y)+\theta_{2}(x) \theta_{3}(x) \theta_{1}(y) \theta_{4}(y), \\
\theta_{2}(x+y) \theta_{3}(x-y) \theta_{2}(0) \theta_{3}(0)=\theta_{2}(x) \theta_{3}(x) \theta_{2}(y) \theta_{3}(y)-\theta_{1}(x) \theta_{4}(x) \theta_{1}(y) \theta_{4}(y),
\end{gathered}
$$




$$
\begin{gathered}
\theta_{2}(x+y) \theta_{4}(x-y) \theta_{2}(0) \theta_{4}(0)=\theta_{2}(x) \theta_{4}(x) \theta_{2}(y) \theta_{4}(y)-\theta_{1}(x) \theta_{3}(x) \theta_{1}(y) \theta_{3}(y), \\
\theta_{3}(x+y) \theta_{4}(x-y) \theta_{3}(0) \theta_{4}(0)=\theta_{3}(x) \theta_{4}(x) \theta_{3}(y) \theta_{4}(y)-\theta_{1}(x) \theta_{2}(x) \theta_{1}(y) \theta_{2}(y), \\
\theta_{2}^{2}(0) \theta_{3}^{2}(x)=\theta_{4}^{2}(0) \theta_{1}^{2}(x)+\theta_{3}^{2}(0) \theta_{2}^{2}(x) \\
\theta_{2}^{2}(0) \theta_{4}^{2}(x)=\theta_{3}^{2}(0) \theta_{1}^{2}(x)+\theta_{4}^{2}(0) \theta_{2}^{2}(x), \\
\theta_{2}^{2}(0) \theta_{1}^{2}(x)=\theta_{3}^{2}(0) \theta_{4}^{2}(x)-\theta_{4}^{2}(0) \theta_{3}^{2}(x) \\
\theta_{2}^{2}(0) \theta_{2}^{2}(x)=\theta_{3}^{2}(0) \theta_{3}^{2}(x)-\theta_{4}^{2}(0) \theta_{4}^{2}(x)
\end{gathered}
$$

They can be proven by rearranging factors in the summation. ${ }^{7,8}$ Two important results for the derivatives of theta functions are $\mathrm{f}^{7,8}$

$$
\theta_{1}^{\prime}(0)=\theta_{2}(0) \theta_{3}(0) \theta_{4}(0), \quad \frac{\theta_{1}^{\prime \prime \prime}(0)}{\theta_{1}^{\prime}(0)}=\frac{\theta_{2}^{\prime \prime}(0)}{\theta_{2}(0)}+\frac{\theta_{3}^{\prime \prime}(0)}{\theta_{3}(0)}+\frac{\theta_{4}^{\prime \prime}(0)}{\theta_{4}(0)}
$$

By considering the quadratic term in the Taylor series of Eqs. (6.9)-(6.11), one obtains

$$
\begin{aligned}
& \frac{\theta_{4}^{\prime \prime}(0)}{\theta_{4}(0)}-\frac{\theta_{3}^{\prime \prime}(0)}{\theta_{3}(0)}=\theta_{2}^{4}(0), \\
& \frac{\theta_{4}^{\prime \prime}(0)}{\theta_{4}(0)}-\frac{\theta_{2}^{\prime \prime}(0)}{\theta_{2}(0)}=\theta_{3}^{4}(0), \\
& \frac{\theta_{3}^{\prime \prime}(0)}{\theta_{3}(0)}-\frac{\theta_{2}^{\prime \prime}(0)}{\theta_{2}(0)}=\theta_{4}^{4}(0), \\
& \theta_{3}^{4}(0)=\theta_{2}^{4}(0)+\theta_{4}^{4}(0) .
\end{aligned}
$$

We introduce the Hirota operator

$$
\begin{gathered}
D_{x}^{m} D_{t}^{n} f . g=\left.\left(\frac{\partial}{\partial x}-\frac{\partial}{\partial x^{\prime}}\right)^{m}\left(\frac{\partial}{\partial t}-\frac{\partial}{\partial t^{\prime}}\right)^{n} f(x, t) g\left(x^{\prime}, t^{\prime}\right)\right|_{x=x^{\prime}, t=t^{\prime}}, \\
D_{x}^{m}(\exp (i \alpha x) g . f)=\left[\left(D_{x}+i \alpha\right)^{m} g . f\right] \exp (i \alpha x) .
\end{gathered}
$$

Differentiating Eq. (6.1) with respect to $y$ and setting $y=0$ yields

$$
D_{x}^{2} \theta_{3}(x) . \theta_{3}(x)=\frac{2 \theta_{2}(0) \theta_{2}^{\prime \prime}(0) \theta_{3}^{2}(x)+2\left(\theta_{1}^{\prime}(0)\right)^{2} \theta_{4}^{2}(x)}{\theta_{2}^{2}(0)}=b_{2} \theta_{3}^{2}(x)+b_{1} \theta_{4}^{2}(x)
$$

Similarly

$$
\begin{gathered}
D_{x}^{2} \theta_{4}(x) . \theta_{4}(x)=b_{1} \theta_{3}^{2}(x)+b_{2} \theta_{4}^{2}(x), \quad D_{x}^{2} \theta_{3}(x) . \theta_{4}(x)=b_{3} \theta_{3}(x) \theta_{4}(x), \\
b_{1}=2 \theta_{3}^{2}(0) \theta_{4}^{2}(0), \quad b_{2}=2 \frac{\theta_{2}^{\prime \prime}(0)}{\theta_{2}(0)}, \quad b_{3}=\frac{\theta_{3}^{\prime \prime}(0)}{\theta_{3}(0)}+\frac{\theta_{4}^{\prime \prime}(0)}{\theta_{4}(0)}
\end{gathered}
$$

Equation (6.3) enables us to write 


$$
\begin{gathered}
D_{x} \theta_{1}(x) \cdot \theta_{2}(x)=\theta_{2}^{2}(0) \theta_{3}(x) \theta_{4}(x) \\
D_{x}^{2} \theta_{1}(x) \cdot \theta_{2}(x)=\left(\frac{\theta_{3}^{\prime \prime}(0)}{\theta_{3}(0)}+\frac{\theta_{4}^{\prime \prime}(0)}{\theta_{4}(0)}\right) \theta_{1}(x) \theta_{2}(x)
\end{gathered}
$$

Thus formulas for $D_{x} \theta_{m}, \theta_{n}, D_{x}^{2} \theta_{m} . \theta_{n}$ can be developed for $m, n$ different using Eqs. (6.3)-(6.8).

${ }^{1}$ N. Akhmediev and A. Ankiewicz, Phys. Rev. A 47, 3213 (1993).

${ }^{2}$ J. P. Boyd, J. Math. Phys. 23, 375 (1982).

${ }^{3}$ J. P. Boyd, Adv. Appl. Mech. 27, 1 (1989).

${ }^{4}$ M. Abramowitz and I. Stegun, Handbook of Mathematical Functions (Dover, New York, 1965).

${ }_{5}^{5}$ D. Lawden, Elliptic Functions and Applications, Applied Mathematical Sciences Vol. 80 (Springer-Verlag, Berlin, 1989).

JJ. D. Fenton and R. S. Gardiner-Garden, J. Austr. Math. Soc. B 24, 47 (1982).

${ }^{7}$ Y. C. Ma, Stud. Appl. Math. 60, 43 (1979).

${ }^{8}$ D. Mihalache, F. Lederer, and D. M. Baboiu, Phys. Rev. A 47, 3285 (1993).

9 A. K. Dhar and K. P. Das, Phys. Fluids A 2, 778 (1990).

${ }^{10}$ A. Hasegawa, Optical Solitons in Fibers (Springer, New York, 1990).

${ }^{11}$ R. Hirota, J. Math. Phys. 14, 805 (1973).

${ }^{12} \mathrm{M}$. Tajiri and Y. Murakami, J. Math. Phys. 34, 2400 (1993).

${ }^{13}$ K. W. Chow, J. Math. Phys. 35, 4059 (1994).

${ }^{14}$ Y. C. Ma and L. G. Redekopp, Phys. Fluids 22, 1872 (1979).

${ }^{15}$ K. W. Chow, J. Phys. Soc. Jpn. 62, 2007 (1993).

${ }^{16} \mathrm{D}$. W. McLaughlin, Important Developments in Soliton Theory, edited by A. S. Fokas and V. E. Zakharov (SpringerVerlag, New York, 1993), p. 537. 